\title{
Lamellar to micellar phases and beyond: when tactic active systems admit free-energy functionals
}

\author{
J. O'Byrne ${ }^{1}$ and J. Tailleur ${ }^{1}$ \\ ${ }^{1}$ Université de Paris, Laboratoire Matière et Systèmes Complexes (MSC), UMR 7057 CNRS, F-75205 Paris, France
}

(Dated: July 9, 2020)

\begin{abstract}
We consider microscopic models of active particles whose velocities, rotational diffusivities, and tumbling rates depend on the gradient of a local field, which is either externally imposed or depends on all particle positions. Despite the fundamental differences between active and passive dynamics at the microscopic scale, we show that a large class of such tactic active systems admit fluctuating hydrodynamics equivalent to those of interacting Brownian colloids in equilibrium. We exploit this mapping to show how taxis may lead to the lamellar and micellar phases observed for soft repulsive colloids. In the context of chemotaxis, we show how the competition between chemoattractant and chemorepellent may lead to a bona-fide equilibrium liquid-gas phase separation in which a loss of thermodynamic stability of the fluid signals the onset of a chemotactic collapse.
\end{abstract}

Over the past ten years, the development of a wealth of synthetic active systems has paved the way for engineering active materials [1-9]. Unlike in equilibrium, however, there is no guiding principle for the self-assembly of active systems, due to the lack of a generic expression for their steady states. A natural way forward, which has been heavily investigated recently [10-17], is then to determine to which extent the knowledge we have garnered in-and close toequilibrium remains relevant to active matter. A first positive answer is provided by the wealth of works on effective temperatures in active systems [18-23] which show that an effective fluctuation-dissipation relation may, under some conditions, survive activity. Then, similarities between active and passive dynamics have also been detected at the level of collective behaviours [24-27]. For instance, motility-induced phase separation (MIPS), which happens at large persistence lengths and times, relies on a feedback between activity and kinetic hindrance without counterparts in thermal equilibrium. Nevertheless, it shares several features, at a coarse-grained level, with an equilibrium liquid-gas phase separation [1013, 28-30]. A natural question is then how general this mesoto-large-scale similarity between active and passive dynamics is, and under which conditions, if any, the microscopic drive out of equilibrium of active particles disappears upon coarsegraining [31].

Among the physical phenomena that control active dynamics, taxis plays an important role in a large range of situations [32]. It is widespread in the biological world, from the chemotaxis of run-and-tumble bacteria [33] to the phototaxis of algae [34] through the durotaxis of cells [35]. Taxis is however not limited to living systems: self-propelled colloids often rely on the presence of chemicals in their environment that not only power their self-propulsion [1] but also bias their dynamics $[36,37]$. Taxis is known to lead to rich, systemspecific behaviours, that occur both at the microscopic and collective levels [36-44]. While some of these phenomena are nonequilibrium in nature [41, 43, 45-47], the aforementioned connections between active systems and equilibrium physics raises the question as to whether microscopic tactic dynamics may also lead to emerging behaviours described by coarse-grained equilibrium theories.

In this Letter, we answer this question for a broad class of active particles, which we refer to as 'tactic active particles' (TAPs), whose propelling speeds or orientational dynamics are biased by the gradients of a field $c(\mathbf{r}, t)$. Using a diffusive scaling of time and space, we construct their fluctuating hydrodynamics and determine under which conditions the latter satisfy detailed balance, whence admitting a free-energy functional. This allows us to reveal a one-to-one correspondance, at this coarse-grained level, between tactic active particles and interacting Brownian colloids. Despite the fundamental differences between these two systems at the microscopic scales, a mapping may thus exist between the large-scale dynamics and steady-state statistics of their density fields. We then exploit this result to report the existence of the micellar and lamellar phases usually observed for softly repulsive Brownian colloids. In the context of chemotaxis, we further show how the competition between chemoattractant and chemorepellent can be rationalized using the physics of Brownian colloids undergoing a liquid-gas phase separation. A simple criterion is then proposed to predict the onset of a chemotactic collapse, which amounts to a loss of thermodynamic stability of the fluid phase. Finally, we show that externally imposed chemical fields $c(\mathbf{r})$ map onto external potentials in equilibrium.

We consider $N$ self-propelled particles whose positions evolve as

$$
\dot{\mathbf{r}}_{i}=v_{p} \mathbf{u}_{i}+\sqrt{2 D_{t}} \boldsymbol{\eta}_{i}
$$

where $\mathbf{u}_{i}$ is a unit vector indicating the orientation of particle $i, v_{p}$ is its self-propulsion speed, and $\left\{\boldsymbol{\eta}_{i}\right\}$ forms a set of $N$ Gaussian white noises whose spatial components satisfy $\left\langle\eta_{i}^{a}(t) \eta_{j}^{b}\left(t^{\prime}\right)\right\rangle=\delta^{a b} \delta_{i j} \delta\left(t-t^{\prime}\right)$. The particles' orientations evolve through rotational diffusion (in $d>1$ dimensions) and instantaneous tumbles. To model taxis, we consider two possible couplings between the field $c$ and the active dynamics. First, the speed of a particle may depend on its orientation with respect to $\nabla c$, through a linear coupling

$$
v_{p}=v_{0}-v_{1} \mathbf{u}_{i} \cdot \nabla c
$$

Alternatively, taxis may stem from the anisotropy of the orientational dynamics. We model the latter as direction-dependent 
tumbling rate $\alpha$ and rotational diffusivity $\Gamma$ [48]:

$$
\alpha=\alpha_{0}+\alpha_{1} \mathbf{u}_{i} \cdot \nabla c \text { and } \Gamma=\Gamma_{0}+\Gamma_{1} \mathbf{u}_{i} \cdot \nabla c
$$

Positive values of $v_{1}, \alpha_{1}$, and $\Gamma_{1}$ drive the particles towards lower values of $c$.

Experimentally, Eq. (2) can be implemented using feedback loops $[49,50]$ when the speed of self-propelled particles can be controlled by light - a class that comprises both Janus colloids $[5,49,51]$ and bacteria [52-55]. The field $c\left(\mathbf{r},\left\{\mathbf{r}_{i}\right\}\right)$ can then be an arbitrary function of all particle positions. Equation (3) is also a standard model for the chemotaxis of runand-tumble bacteria $[33,40,46,56]$. The field $c(\mathbf{r})$ then models chemorepellent $\left(\alpha_{1}>0\right)$ or chemoattractant $\left(\alpha_{1}<0\right)$, which can be either produced by the bacteria or imposed externally. To cover both cases, we consider the many-body dynamics in which $c\left(\mathbf{r}_{i},[\rho]\right)$ is both a function of $\mathbf{r}_{i}$ and a functional of the particle density. In the context of diffusing fields, this amounts to integrating out the dynamics of the chemotactic fields, following, e.g., [36, 37].

Fluctuating mesoscopic description. While much insight can be gained from deterministic hydrodynamic descriptions of active systems [46, 57-59], measuring their irreversibility by, say, computing entropy production requires working at the fluctuating level. At scales much larger than the persistence length of the particles, we expect the active random walks to lead to a diffusive behaviour. We thus rescale space and time as $(x, t) \rightarrow\left(x / L, t / L^{2}\right)$, where $L$ is the linear size of the system. To lighten the notations, we focus for now on rotational diffusion. The $N$-body probability density $\psi\left(\left\{\mathbf{r}_{i}, \mathbf{u}_{i}\right\}, t\right)$ then evolves as

$$
\begin{aligned}
\partial_{t} \psi= & -L \sum_{i=1}^{N} \nabla_{\mathbf{r}_{i}} \cdot\left[\left(v_{0}-\frac{v_{1}}{L} \mathbf{u}_{i} \cdot \nabla_{\mathbf{r}_{i}} c\right) \mathbf{u}_{i} \psi-\frac{D_{t}}{L} \nabla_{\mathbf{r}_{i}} \psi\right] \\
& +L^{2} \sum_{i} \Delta_{\mathbf{u}_{i}}\left[\left(\Gamma_{0}+\frac{\Gamma_{1}}{L} \mathbf{u}_{i} \cdot \nabla_{\mathbf{r}_{i}} c\right) \psi\right]
\end{aligned}
$$

Integrating over all orientational degrees of freedom leads to a conservation equation $\partial_{t} \phi\left(\left\{\mathbf{r}_{i}\right\}, t\right)=-\sum_{i} \nabla_{\mathbf{r}_{i}} \cdot \mathbf{J}_{i}\left(\left\{\mathbf{r}_{i}\right\}, t\right)$, where $\mathbf{J}_{i} \equiv-D_{t} \nabla_{\mathbf{r}_{i}} \phi+\int \Pi_{j} \mathrm{~d} \mathbf{u}_{j}\left(v_{0} L-v_{1} \mathbf{u}_{i} \cdot \nabla_{\mathbf{r}_{i}} c\right) \mathbf{u}_{i} \psi$ is the probability current along direction $\mathbf{r}_{i}$ and $\phi$ is the marginal in space of $\psi$. To compute the diffusive limit of $\mathbf{J}_{i}$, the dynamics (4) can be projected onto its successive $N$-body harmonics, leading to a hierarchy of equations [60]. Under the asumption of a lack of long-range correlations between the particle orientations, a closure is obtained by noticing that all moments beyond $\phi\left(\left\{\mathbf{r}_{i}\right\}, t\right)$ are fast fields and decay as $1 / L$ (or faster) in the large system-size limit, leading to:

$$
\begin{aligned}
\mathbf{J}_{i} \simeq & -\left(D_{t}+\frac{v_{0}^{2}}{d(d-1) \Gamma_{0}}\right) \nabla_{\mathbf{r}_{i}} \phi-\phi\left[\frac{\nabla_{\mathbf{r}_{i}} v_{0}^{2}}{2 d(d-1) \Gamma_{0}}\right. \\
& \left.+\left(\frac{v_{0} \Gamma_{1}+v_{1} \Gamma_{0}}{d \Gamma_{0}}\right) \nabla_{\mathbf{r}_{i}} c\right]
\end{aligned}
$$

At this stage, we have constructed a coarse-grained diffusive description of dynamics (1)-(3). We now restore, for full generality, the possibility of tumbles and take $v_{i}, \alpha_{i}$, and $\Gamma_{i}$ constant to focus on the consequences of taxis. Using stochastic calculus, one then obtains a corresponding fluctuating hydrodynamics for the density field $\rho(\mathbf{r})=\sum_{i=1}^{N} \delta\left(\mathbf{r}-\mathbf{r}_{i}\right)$ :

$$
\begin{aligned}
\partial_{t} \rho & =-\nabla \cdot\left[J_{D}+\sqrt{2 D \rho} \Lambda\right] \\
J_{D} & =-D \rho \nabla\left[\log \rho+\left(v_{1}+v_{0} \frac{\alpha_{1}+(d-1) \Gamma_{1}}{\alpha_{0}+(d-1) \Gamma_{0}}\right) \frac{c}{d D}\right](7)
\end{aligned}
$$

where $\Lambda(\mathbf{r}, t)$ is a Gaussian noise field of zero mean and correlations $\left\langle\Lambda(\mathbf{r}, t) \Lambda\left(\mathbf{r}^{\prime}, t^{\prime}\right)\right\rangle=\delta\left(t-t^{\prime}\right) \delta\left(\mathbf{r}-\mathbf{r}^{\prime}\right), J_{D}$ is the deterministic part of the current, and $D$ is a large-scale diffusivity:

$$
D=D_{t}+\frac{v_{0}^{2}}{d\left(\alpha_{0}+(d-1) \Gamma_{0}\right)} .
$$

Equations (6) and (7) describe the stochastic dynamics of the density field of TAPs at scales much larger than their persistence lengths and times, and can now be used to study their emerging collective behaviours.

Effective free-energy functionals. Inspection of Eq. (7) shows that the deterministic part of the current can be written as $J_{D}=-D \rho \nabla \mu$, where $\mu$ plays the role of a nonequilibrium chemical potential [61]. An interesting outcome of the diffusive scaling is that the noise field and the mobility appearing in $J_{D}$ satisfy a generalized Stokes-Einstein relation. The dynamics (6) then satisfies detailed balance whenever one can find a free energy $\mathcal{F}[\rho]$ whose functional derivative is given by $\mu$. The solution of such an inverse variational problem [62] can be obtained by generalizing the Schwarz condition of integrability to functional integration. In practice, we introduce

$$
\mathcal{D}\left(\mathbf{r}, \mathbf{r}^{\prime}\right)=\frac{\delta \mu([\rho], \mathbf{r})}{\delta \rho\left(\mathbf{r}^{\prime}\right)}-\frac{\delta \mu\left([\rho], \mathbf{r}^{\prime}\right)}{\delta \rho(\mathbf{r})},
$$

which is such that $\mu(\mathbf{r},[\rho])$ is a functional derivative iif, for any two test functions $f, g$ [63],

$$
\int \mathcal{D}\left(\mathbf{r}, \mathbf{r}^{\prime}\right) f(\mathbf{r}) g\left(\mathbf{r}^{\prime}\right) \mathrm{d} \mathbf{r} \mathrm{d} \mathbf{r}^{\prime}=0 .
$$

Note that Eq. (10) means that $\mathcal{D}$ vanishes as a distribution, which is not always straightforward to read in its expression. For instance, in one dimension, $\mu=\partial_{x}^{k} \rho$ leads to $\mathcal{D}\left(x, x^{\prime}\right)=$ $\left(\partial_{x}^{k}-\partial_{x^{\prime}}^{k}\right) \delta\left(x-x^{\prime}\right) ; \mu$ admits a functional integral iif $k$ is even, which can be checked easily using (10).

Let us now consider a field $c$ given by:

$$
c([\rho], \mathbf{r})=\int K\left(\mathbf{r}, \mathbf{r}_{1}, \ldots, \mathbf{r}_{p-1}\right) \rho\left(\mathbf{r}_{1}\right) \ldots \rho\left(\mathbf{r}_{p-1}\right) \mathrm{d} \mathbf{r}_{1} \ldots \mathrm{d} \mathbf{r}_{p-1}
$$

Equation (10) is satisfied whenever the kernel $K$ is invariant under any permutation of its variables. The fluctuating hydrodynamics (6)-(7) then becomes:

$$
\partial_{t} \rho=\nabla \cdot\left[D \rho \nabla \frac{\delta \mathcal{F}}{\delta \rho}+\sqrt{2 D \rho} \Lambda\right]
$$



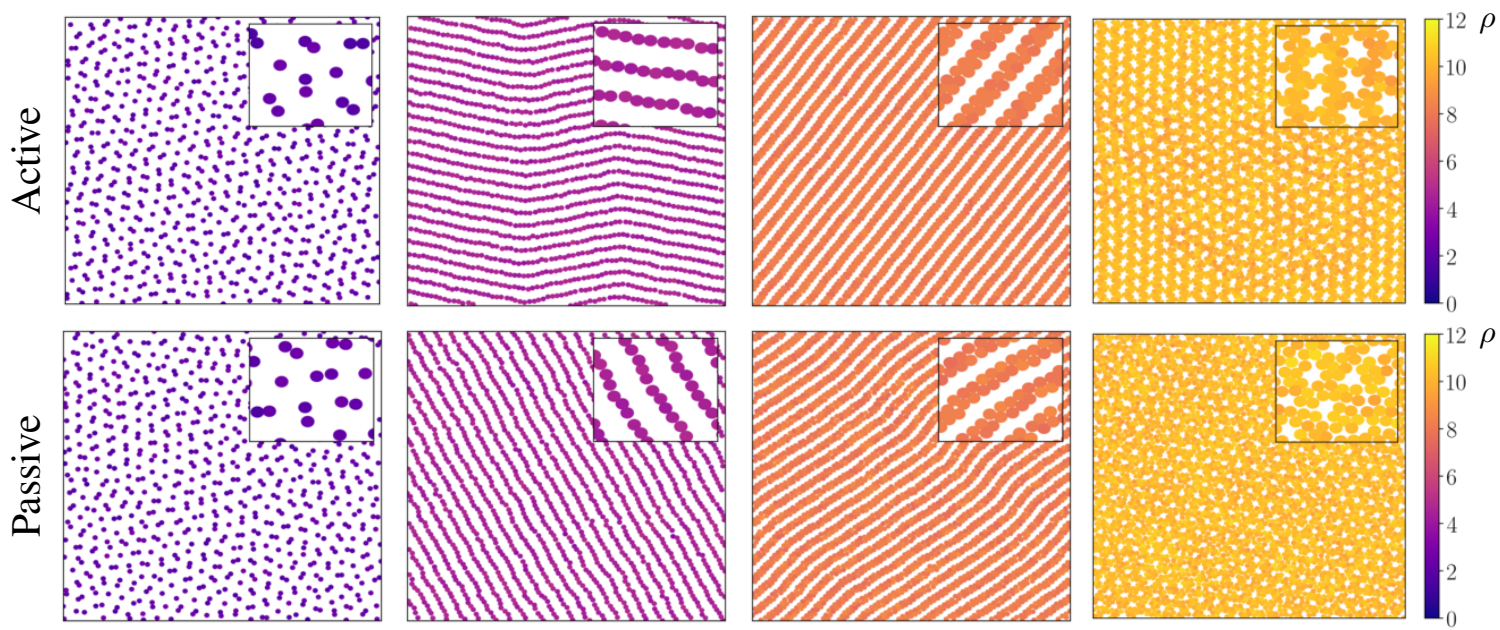

FIG. 1. Simulations of RTP dynamics (1)-(2) (top) and of the equilibrium dynamics (14) (bottom), under the conditions of the mapping (15) with $K(\mathbf{r})$ defined by (16). Color encodes the local density. Parameters: $v_{0}=1, v_{1}=0.2, \alpha_{0}=50, D_{t}=0, \sigma_{0}=0.3, \sigma_{1}=1, \varepsilon=5$, $E=10, d t=10^{-3}$. Snapshots taken at $t=20000$ in a system of size $20 \times 20$. From left to right, $\rho_{0} \equiv N / L^{2}$ is equal to $2,4,8$ and 10 .

where the effective free-energy functional $\mathcal{F}$ is given by:

$$
\begin{aligned}
\mathcal{F}[\rho]= & \int \mathrm{d} \mathbf{r} \rho(\mathbf{r}) \log \rho(\mathbf{r})+\frac{v_{0}}{d D}\left[\frac{v_{1}}{v_{0}}+\frac{\alpha_{1}+(d-1) \Gamma_{1}}{\alpha_{0}+(d-1) \Gamma_{0}}\right] \times \\
& \frac{1}{p !} \int K\left(\mathbf{r}_{1}, \ldots, \mathbf{r}_{p}\right) \rho\left(\mathbf{r}_{1}\right) \ldots \rho\left(\mathbf{r}_{p}\right) \mathrm{d} \mathbf{r}_{1} \ldots \mathrm{d} \mathbf{r}_{p}
\end{aligned}
$$

Importantly, Eqs. (12) and (13) also describe the fluctuating hydrodynamics of $N$ Brownian colloids interacting via a $p$ body potential. More precisely, consider $N$ particles undergoing the equilibrium Langevin dynamics

$$
\gamma \dot{\mathbf{r}}_{i}=-\sum_{j_{1}<\ldots<j_{p-1}} \nabla_{\mathbf{r}_{i}} V\left(\mathbf{r}_{i}, \mathbf{r}_{j_{1}}, \ldots, \mathbf{r}_{j_{p-1}}\right)+\sqrt{2 \gamma T} \boldsymbol{\eta}
$$

where we have introduced a temperature $T$, a damping $\gamma$ and a $p$-body potential $V$. Equations (12) and (13) describe the fluctuating hydrodynamics of this system, upon identifying:

$$
\begin{aligned}
\gamma^{-1} T & \Leftrightarrow D \\
\gamma^{-1} V\left(\mathbf{r}_{1}, \ldots, \mathbf{r}_{p}\right) & \Leftrightarrow\left[\frac{v_{1}}{d}+\frac{v_{0}}{d} \frac{\alpha_{1}+(d-1) \Gamma_{1}}{\alpha_{0}+(d-1) \Gamma_{0}}\right] K\left(\mathbf{r}_{1}, \ldots, \mathbf{r}_{p}\right) .
\end{aligned}
$$

The mapping (15) establishes a macroscopic connection between the non-equilibrium dynamics (1)-(3) and the equilibrium dynamics (14), which strongly differs at the microscopic scale. In particular, the roles played by $K$ and $V$ in these microscopic dynamics are of very different natures, despite their similar role in the macroscopic dynamics of $\rho$. This differs from approaches in which the chemotaxis of cells is already modelled at the microscopic level by Brownian dynamics in which the field $c$ plays the role of a potential [64]. The macroscopic equivalence described there with equilibrium dynamics directly stems from a microscopic one. Note that, while a similarity between active and passive collective behaviours has been reported in the case of MIPS [10-13, 28-30], the mapping (15) suggests a much broader relationship between passive and active systems, which we now explore.

Micellar, lamellar and crystalline phases. The recent development of active systems in which the particle velocities can be controlled individually using light and feedback loops [49, 50] makes the realization of dynamics (1)-(2) within experimental reach. To explore and rationalize the physics these systems can access, we consider a simple case in which the field $c$ is obtained using a convolution kernel $K\left(\mathbf{r}, \mathbf{r}_{1}\right)=K\left(\mathbf{r}-\mathbf{r}_{1}\right)$ with two typical lengthscales:

$$
K(\mathbf{r})=A \mathrm{e}^{-\frac{\sigma_{0}^{2}}{\sigma_{0}^{2}-r^{2}}} \Theta\left(\sigma_{0}-|\mathbf{r}|\right)+\epsilon \mathrm{e}^{-\frac{\sigma_{1}^{2}}{\sigma_{1}^{2}-r^{2}}} \Theta\left(\sigma_{1}-|\mathbf{r}|\right),
$$

where $\Theta(u)$ is the Heaviside function and we consider $A>\epsilon$ and $\sigma_{1}>\sigma_{0}$. We report in the top row of Fig. 1 simulations of the active dynamics (1)-(2). As the density increases, the system undergoes a series of phase transitions: a disordered gas (not shown) is first replaced by a crystal of 'micelles', each comprising an increasing number of particles as the density increases $\left(1^{\text {st }}\right.$ column$)$, until a laning transition occurs. Further increasing the density increases the local number of particles forming the lanes $\left(2^{\text {nd }} \& 3^{\text {rd }}\right.$ columns $)$. At higher densities, inverted crystals develop, in which voids and particles have exchanged their previous roles $\left(4^{\text {th }}\right.$ column). The underlying physics can be rationalized thanks to our equilibrium mapping: simulations of the passive dynamics (14) for parameters satisfying (15) indeed perfectly match those of TAPs (Fig. 1, bottom row). In the passive picture, the kernel (16) corresponds to a pairwise potentials with a repulsive central core and a softer repulsive shoulder. Using equilibrium Monte Carlo simulations, similar potentials have been shown to lead to a variety of exotic phases [65-67], which are thus accessi- 


\section{ble to TAPS.}

Chemotaxis: competition between attraction and repulsion. While fields $c(\mathbf{r},[\rho])$ given by (11) can be engineered in the lab, they can also be found in nature. In biological systems, tactic interactions are often mediated by diffusing molecules, which naturally lead to a linear coupling between $\rho$ and $c$. Interactions mediated by fluctuating membranes or interfaces would lead to non-linearities which offer an interesting (and challenging) problem left for future works. Inspired by bacterial chemotaxis, we consider a model system in which active particles interact through the production of a chemoattractant and a chemorepellent. Introducing their concentration fields $c_{\mathrm{a}}$ and $c_{\mathrm{r}}$, we write the tumbling rate of particle $i$ as $\alpha=\alpha_{0}-\alpha_{1}^{\mathrm{a}} \mathbf{u}_{i} \cdot \nabla c_{\mathrm{a}}+\alpha_{1}^{\mathrm{r}} \mathbf{u}_{i} \cdot \nabla c_{\mathrm{r}}$. Taking $\alpha_{1}^{\mathrm{r}}>0$ and $\alpha_{1}^{\mathrm{a}}>0$ then bias the random walk of particle $i$ towards high- $c_{\mathrm{a}}$ regions and away from high- $c_{\mathrm{r}}$ regions.

After their production by the particles, at rates $a_{\mathrm{a}, \mathrm{r}}$, the signaling molecules diffuse with diffusivity $\nu_{\mathrm{a}, \mathrm{r}}$ and are degraded at rates $\lambda_{\mathrm{a}, \mathrm{r}}$, leading to the dynamics

$$
\partial_{t} c_{\mathrm{n}}(\mathbf{r})=\nu_{\mathrm{n}} \Delta c_{\mathrm{n}}(\mathbf{r})-\lambda_{\mathrm{n}} c_{\mathrm{n}}+a_{\mathrm{n}} \sum_{i} \delta\left(\mathbf{r}-\mathbf{r}_{i}\right)
$$

where $n \in\{\mathrm{a}, \mathrm{r}\}$. A standard, fast variable treatment on $c_{\mathrm{n}}$ then leads to the screened Poisson equation

$$
\left(\Delta-\frac{\lambda_{\mathrm{n}}}{\nu_{\mathrm{n}}}\right) c_{\mathrm{n}}=-\frac{a_{\mathrm{n}}}{\nu_{\mathrm{n}}} \rho,
$$

which can be solved as $c_{\mathrm{n}}=\frac{a_{\mathrm{n}}}{\nu_{\mathrm{n}}} G_{\mathrm{n}} * \rho$, where $G_{\mathrm{n}}$ is the Green function of Eq. (18). The system thus satisfies the mapping condition (11) with $p=2$. Introducing the screening lengths $\ell_{\mathrm{n}} \equiv \sqrt{\nu_{\mathrm{n}} / \lambda_{\mathrm{n}}}$, the Green functions are given by $G_{\mathrm{n}}(\mathbf{r})=\ell_{\mathrm{n}} e^{-|\mathbf{r}| / \ell_{\mathrm{n}}} / 2$ in $1 \mathrm{D}, G_{\mathrm{n}}(\mathbf{r})=K_{0}\left(|\mathbf{r}| / \ell_{\mathrm{n}}\right) / 2 \pi$ in $2 \mathrm{D}$, where $K_{0}$ is the $0^{t h}$ order modified Bessel function of the second kind, and $K(\mathbf{r})=e^{-|\mathbf{r}| / \ell_{\mathrm{n}}} / 4 \pi|\mathbf{r}|$ in $3 \mathrm{D}$. The mapping (15) then shows this system of active particles to be equivalent, at the fluctuating hydrodynamic level, to passive Brownian particles interacting via a pair potential

$$
V(\mathbf{r})=\frac{\gamma v_{0}}{d \alpha_{0}}\left[\alpha_{1}^{\mathrm{r}} \frac{a_{\mathrm{r}}}{\nu_{\mathrm{r}}} G_{\mathrm{r}}(\mathbf{r})-\alpha_{1}^{\mathrm{a}} \frac{a_{\mathrm{a}}}{\nu_{\mathrm{a}}} G_{\mathrm{a}}(\mathbf{r})\right] .
$$

The superposition of chemoattractant and chemorepellent thus directly maps onto an equilibrium problem with attractive and repulsive interactions. Let us consider the case in which $\alpha_{1}^{\mathrm{r}}>\alpha_{1}^{\mathrm{a}}$ and $\ell_{\mathrm{r}} \leq \ell_{\mathrm{a}}$. The physics of this chemotactic system is now mapped onto the well-known problem of repulsive hard-core interactions with attractive tails. The system is purely repulsive for $\ell_{\mathrm{a}}=\ell_{\mathrm{r}}$, hence leading to a gas phase. As $\ell_{\mathrm{r}}$ decreases, the attractive tail develops, allowing for a liquid-gas coexistence. For even shorter $\ell_{\mathrm{r}}$, the liquid phase becomes thermodynamically unstable leading to a collapse of the system when $\bar{V} \equiv \int \mathrm{d} \mathbf{r} V(\mathbf{r})<0$ [68]. Figure 2 compares simulations of the passive dynamics (14) and the active dynamics (1)-(3), using the 2D Green functions of Eq. (18), as $\ell_{\mathrm{r}}$ is varied. Once again, despite the fundamental differences between their microscopic dynamics and interactions,
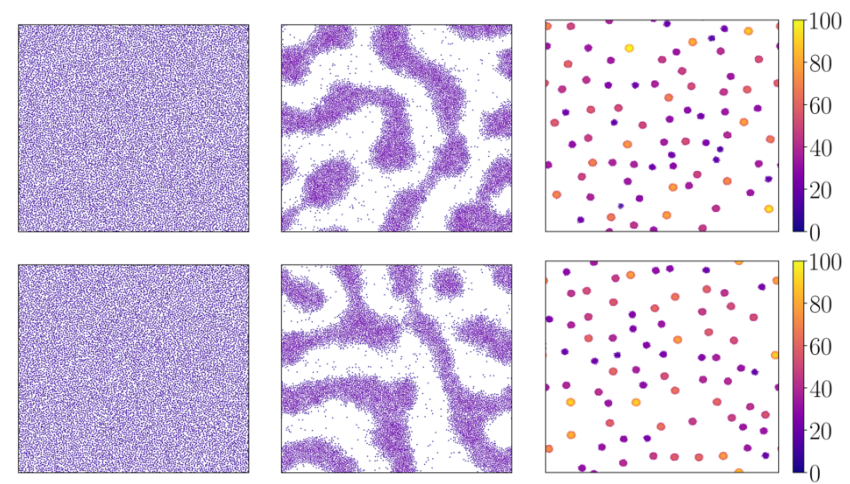

FIG. 2. Simulations of the active dynamics (1)-(3), top, and the equilibrium dynamics (14), bottom, using the pair-potential (19), under the conditions of the mapping (15). Color encodes the local density. From left to right $\ell_{\mathrm{r}}=1 / 6,1 / 8,1 / 10$. A chemotactic collapse is predicted whenever $\bar{V}<0$, i.e. below $\ell_{\mathrm{r}}=1 / 8.2$. Parameters: $L=40, \rho_{0}=7, a_{\mathrm{a}} / 2 \pi \nu_{\mathrm{a}}=0.05, \ell_{\mathrm{a}}=0.25, a_{\mathrm{r}} / 2 \pi \nu_{\mathrm{r}}=0.2$, $\alpha_{0}=100, \alpha_{1}^{\mathrm{r}}=\alpha_{1}^{\mathrm{a}}=200, v_{0}=1, T^{-1}=200, \gamma=1$, $\mathrm{d} t=10^{-3}$. The snapshots were taken after a time $t=10^{4}$.

the large-scale physics of these two systems are hardly distinguishable.

External fields. Finally, while we have focused so far on collective effects, nothing prevents an additional taxis towards an externally controled field $w(\mathbf{r})$. Our models of taxis (2) and (3) are easily generalized to this case, using for instance:

$$
\left\{\begin{array}{l}
v_{p}=v_{0}-v_{1} \mathbf{u}_{i} \cdot \nabla c-v_{2} \mathbf{u}_{i} \cdot \nabla w \\
\alpha=\alpha_{0}+\alpha_{1} \mathbf{u}_{i} \cdot \nabla c+a_{2} \mathbf{u}_{i} \cdot \nabla w \\
\Gamma=\Gamma_{0}+\Gamma_{1} \mathbf{u}_{i} \cdot \nabla c+\Gamma_{2} \mathbf{u}_{i} \cdot \nabla w
\end{array}\right.
$$

where the field $c$ still accounts for interactions between the self-propelled particles given by Eq. (11). The equilibrium mapping still holds, with a free energy now given by:

$$
\tilde{\mathcal{F}}[\rho]=\mathcal{F}[\rho]+\frac{v_{0}}{d D}\left[\frac{v_{2}}{v_{0}}+\frac{\alpha_{2}+(d-1) \Gamma_{2}}{\alpha_{0}+(d-1) \Gamma_{0}}\right] \int \mathrm{d} \mathbf{r} w(\mathbf{r}) \rho(\mathbf{r})
$$

where $\mathcal{F}$ remains given by Eq. (13). The external field $w(\mathbf{r})$ is thus equivalent to an external potential for Brownian colloids. Much like gravity, it can be used to localize a dense phase in a phase-separated system.

Discussion. The mapping between TAPS and Brownian colloids presented in this article offers an interesting route to control synthetic active matter, for instance to prepare a desired initial condition in a light-controlled active system $[49,50]$. It also offers a qualitative insight into the largescale behaviours of tactic active particles, which, as for MIPS, should hold beyond the sole systems that will obey exactly the dynamics (1)-(3).

The derivation of Eq. (5), detailed in SI [60], works directly at the interacting $N$-body level. This differs from standard treatment of chemotaxis that rely on an explicit dynamics of the field $c$, assumed to be slow, to work at an essentially noninteracting level [69]. Our treatment relies on a lack of longrange orientational order and precludes, in particular, largescale collective motion. Whether the latter may emerge in a 
setting as simple as the one described by (1)- (3) is an open challenging question, stimulated in particular by the works on bacterial travelling waves $[41,46]$. Furthermore, our derivation assumes that the field gradients remain finite, which has to be checked self-consistently. In particular, it certainly does not hold in the late-stage dynamics of the chemotactic collapse reported in Fig. 2, which only highlights the remarkable agreement between passive and active dynamics in this case.

In this Letter, we have focused on active systems whose large-scale dynamics obey detailed balance, but the interest of our mapping is not limited to these cases. Studying the linear response to fields violating (10) or (15) is a natural next step. Near-equilibrium studies of active matter models have indeed been shown to capture many interesting properties of active systems [70-73]. Furthermore, the approach to the inverse variational problem described in Eq. (9)-(10) can easily be generalized to more complex situations, for instance involving several species of active particles [43, 74-76].

Finally, we have focused on the consequences that the mapping (15) bears on the steady-state distributions of tactic active systems. The one-to-one correspondence is, however, established at a dynamical level which paves the way to a study of the transition states of tactic active systems, using methods like transition path sampling [77] or the string method [78].

[1] J. R. Howse, R. A. Jones, A. J. Ryan, T. Gough, R. Vafabakhsh, and R. Golestanian, Physical Review Letters 99, 048102 (2007).

[2] J. Palacci, C. Cottin-Bizonne, C. Ybert, and L. Bocquet, Physical Review Letters 105, 088304 (2010).

[3] S. Thutupalli, R. Seemann, and S. Herminghaus, New Journal of Physics 13, 073021 (2011).

[4] A. Bricard, J.-B. Caussin, N. Desreumaux, O. Dauchot, and D. Bartolo, Nature 503, 95 (2013).

[5] J. Palacci, S. Sacanna, A. P. Steinberg, D. J. Pine, and P. M. Chaikin, Science 339, 936 (2013).

[6] D. Nishiguchi and M. Sano, Physical Review E 92, 052309 (2015).

[7] J. Yan, M. Han, J. Zhang, C. Xu, E. Luijten, and S. Granick, Nature materials 15, 1095 (2016).

[8] C. Bechinger, R. Di Leonardo, H. Löwen, C. Reichhardt, G. Volpe, and G. Volpe, Reviews of Modern Physics 88, 045006 (2016).

[9] P. G. Moerman, H. W. Moyses, E. B. Van Der Wee, D. G. Grier, A. Van Blaaderen, W. K. Kegel, J. Groenewold, and J. Brujic, Physical Review E 96, 032607 (2017).

[10] J. Tailleur and M. Cates, Physical Review Letters 100, 218103 (2008).

[11] T. Speck, J. Bialké, A. M. Menzel, and H. Löwen, Physical Review Letters 112, 218304 (2014).

[12] S. C. Takatori, W. Yan, and J. F. Brady, Physical Review Letters 113, 028103 (2014).

[13] F. Ginot, I. Theurkauff, D. Levis, C. Ybert, L. Bocquet, L. Berthier, and C. Cottin-Bizonne, Physical Review X 5, 011004 (2015).

[14] U. M. B. Marconi, C. Maggi, and S. Melchionna, Soft Matter 12, 5727 (2016).
[15] J. Rodenburg, M. Dijkstra, and R. van Roij, Soft matter 13, 8957 (2017).

[16] A. P. Solon, J. Stenhammar, M. E. Cates, Y. Kafri, and J. Tailleur, Physical Review E 97, 020602 (2018).

[17] E. Flenner and G. Szamel, arXiv preprint arXiv:2004.11925 (2020).

[18] D. Loi, S. Mossa, and L. F. Cugliandolo, Physical Review E 77, 051111 (2008).

[19] S. Wang and P. G. Wolynes, The Journal of Chemical Physics 135, 051101 (2011).

[20] D. Loi, S. Mossa, and L. F. Cugliandolo, Soft Matter 7, 3726 (2011).

[21] K. I. Morozov and L. M. Pismen, Physical Review E 81, 061922 (2010).

[22] G. Szamel, Physical Review E 90, 012111 (2014).

[23] A. P. Solon, M. Cates, and J. Tailleur, The European Physical Journal Special Topics 224, 1231 (2015).

[24] P. Chavanis, M. Ribot, C. Rosier, and C. Sire, Banach Center Publ. 66, 287 (2004).

[25] R. Golestanian, Physical Review Letters 108, 038303 (2012).

[26] S. C. Takatori and J. F. Brady, Physical Review E 91, 032117 (2015).

[27] S. Paliwal, J. Rodenburg, R. van Roij, and M. Dijkstra, New Journal of Physics 20, 015003 (2018).

[28] A. G. Thompson, J. Tailleur, M. E. Cates, and R. A. Blythe, Journal of Statistical Mechanics: Theory and Experiment 2011, P02029 (2011).

[29] D. Bi, X. Yang, M. C. Marchetti, and M. L. Manning, Physical Review X 6, 021011 (2016).

[30] T. Grafke, M. E. Cates, and E. Vanden-Eijnden, Physical Review Letters 119, 188003 (2017).

[31] C. Nardini, É. Fodor, E. Tjhung, F. Van Wijland, J. Tailleur, and M. E. Cates, Physical Review X 7, 021007 (2017).

[32] Taxis describes the biased motion of an entity in response to an external signal.

[33] H. C. Berg, E. coli in Motion (Springer Science \& Business Media, 2008).

[34] M. Polin, I. Tuval, K. Drescher, J. P. Gollub, and R. E. Goldstein, Science 325, 487 (2009).

[35] R. Sunyer, V. Conte, J. Escribano, A. Elosegui-Artola, A. Labernadie, L. Valon, D. Navajas, J. M. García-Aznar, J. J. Muñoz, P. Roca-Cusachs, et al., Science 353, 1157 (2016).

[36] R. Soto and R. Golestanian, Physical Review Letters 112, 068301 (2014).

[37] O. Pohl and H. Stark, Physical Review Letters 112, 238303 (2014).

[38] D. Woodward, R. Tyson, M. Myerscough, J. Murray, E. Budrene, and H. Berg, Biophysical journal 68, 2181 (1995).

[39] M. P. Brenner, L. S. Levitov, and E. O. Budrene, Biophysical journal 74, 1677 (1998).

[40] S. Chatterjee, R. A. da Silveira, and Y. Kafri, PLoS computational biology 7 (2011).

[41] J. Saragosti, V. Calvez, N. Bournaveas, B. Perthame, A. Buguin, and P. Silberzan, Proceedings of the National Academy of Sciences 108, 16235 (2011).

[42] S. Saha, R. Golestanian, and S. Ramaswamy, Physical Review E 89, 062316 (2014).

[43] J. Agudo-Canalejo and R. Golestanian, Physical Review Letters 123, 018101 (2019).

[44] B. Nasouri and R. Golestanian, Physical Review Letters 124, 168003 (2020).

[45] R. E. Goldstein, Physical Review Letters 77, 775 (1996).

[46] M. Seyrich, A. Palugniok, and H. Stark, New Journal of Physics 21, 103001 (2019). 
[47] S. Mahdisoltani, R. B. A. Zinati, C. Duclut, A. Gambassi, and R. Golestanian, arXiv preprint arXiv:1911.08115 (2019).

[48] The rotational diffusion is understood as an Itō-Langevin process.

[49] T. Bäuerle, A. Fischer, T. Speck, and C. Bechinger, Nature communications 9, 1 (2018).

[50] F. A. Lavergne, H. Wendehenne, T. Bäuerle, and C. Bechinger, Science 364, 70 (2019).

[51] I. Buttinoni, J. Bialké, F. Kümmel, H. Löwen, C. Bechinger, and T. Speck, Physical Review Letters 110, 238301 (2013).

[52] G. Vizsnyiczai, G. Frangipane, C. Maggi, F. Saglimbeni, S. Bianchi, and R. Di Leonardo, Nature communications 8 , 1 (2017).

[53] G. Frangipane, D. Dell'Arciprete, S. Petracchini, C. Maggi, F. Saglimbeni, S. Bianchi, G. Vizsnyiczai, M. L. Bernardini, and R. Di Leonardo, Elife 7, e36608 (2018).

[54] J. Arlt, V. A. Martinez, A. Dawson, T. Pilizota, and W. C. Poon, Nature communications 9, 1 (2018).

[55] J. Arlt, V. A. Martinez, A. Dawson, T. Pilizota, and W. C. Poon, Nature communications 10, 1 (2019).

[56] M. J. Schnitzer, Physical Review E 48, 2553 (1993).

[57] E. Bertin, M. Droz, and G. Grégoire, Physical Review E 74, 022101 (2006).

[58] B. Liebchen, D. Marenduzzo, and M. E. Cates, Physical Review Letters 118, 268001 (2017).

[59] M. Kourbane-Houssene, C. Erignoux, T. Bodineau, and J. Tailleur, Physical Review Letters 120, 268003 (2018).

[60] See Supplemental Material [url], which includes theoretical and numerical details, as well as Refs. XXX.

[61] P. M. Chaikin, T. C. Lubensky, and T. A. Witten, Principles of condensed matter physics, Vol. 10 (Cambridge university press Cambridge, 1995).

[62] I. M. Anderson, The variational bicomplex, Tech. Rep. (Utah State Technical Report, 1989, http://math. usu. edu/ fg mp, 1989).

[63] As usual, there are topological requirement for the conditions to be sufficient [62].

[64] P.-H. Chavanis, Communications in Nonlinear Science and Numerical Simulation 15, 60 (2010).

[65] G. Malescio and G. Pellicane, Nature materials 2, 97 (2003).

[66] M. A. Glaser, G. M. Grason, R. D. Kamien, A. Košmrlj, C. D. Santangelo, and P. Ziherl, EPL (Europhysics Letters) 78, 46004 (2007).

[67] H. Löwen, in Understanding Soft Condensed Matter Via Modeling And Computation (World Scientific, 2011) pp. 9-45.

[68] D. Ruelle, Statistical mechanics: Rigorous results (World Scientific, 1999).

[69] H. G. Othmer and T. Hillen, SIAM Journal on Applied Mathematics 61, 751 (2000).

[70] U. M. B. Marconi, N. Gnan, M. Paoluzzi, C. Maggi, and R. Di Leonardo, Scientific reports 6, 1 (2016).

[71] É. Fodor, C. Nardini, M. E. Cates, J. Tailleur, P. Visco, and F. van Wijland, Physical Review Letters 117, 038103 (2016).

[72] R. Wittmann, C. Maggi, A. Sharma, A. Scacchi, J. M. Brader, and U. M. B. Marconi, arXiv preprint arXiv:1701.09032 (2017).

[73] R. Wittmann, U. M. B. Marconi, C. Maggi, and J. M. Brader, arXiv preprint arXiv:1702.00337 (2017).

[74] R. Wittkowski, J. Stenhammar, and M. E. Cates, New Journal of Physics 19, 105003 (2017).

[75] A. I. Curatolo, N. Zhou, Y. Zhao, C. Liu, A. Daerr, J. Tailleur, and J.-D. Huang, BioRxiv , 798827 (2019).

[76] T. Kolb and D. Klotsa, Soft Matter 16, 1967 (2020).

[77] C. Dellago, P. G. Bolhuis, F. S. Csajka, and D. Chandler, The
Journal of chemical physics 108, 1964 (1998).

[78] E. Weinan, W. Ren, and E. Vanden-Eijnden, Physical Review B 66, 052301 (2002). 